\title{
The Mediating Role of Stress in Cultural Differences in Depression: A Comparison of US American and Chinese Culture
}

\author{
Wendi Jia ${ }^{1,2}$ \\ ${ }^{1}$ School of Information Engineering, Nanjing University of Finance \& Economics, Nanjing, Jiangsu 210046, China \\ ${ }^{2}$ School of Computing, National University of Singapore, Singapore 117417, Singapore
}

\begin{abstract}
The current study examined the associations between depression and several potential predictors: intolerance of uncertainty (IU), mindfulness and stress between two different cultures (US America and China). US American participants reported higher levels of depression and stress compared to their Chinese counterparts, and stress was strongly and positively associated with depression for both cultural groups. These results suggest that stress fully mediates the cultural difference in depression. Contrary to our hypotheses, however, there were no cultural differences in IU nor mindfulness. These findings have implications for counseling practice and future research.
\end{abstract}

Keywords: depression, $I U$, mindfulness, stress, culture

\section{INTRODUCTION}

Depressed feelings are always anchored in sadness, gloom, and emotional dejection, while clinical depression is characterized by low moods, sense of hopelessness and meaninglessness, negative self-concept, and increased sadness that lasts over weeks or months and interferes with one's normal functions [1] (Johnson \& Indvik, 1997). Studies have shown that depression rates vary from country to country, and the United States is the leading country in depression rates among 192 countries [2] (World Health Organization [WHO], 2004). By contrast, the depression rate in China is much lower. We suspected that this difference can be attributed to a few potential factors. Based on previous research, John and Indvik (1997) indicated that there are several different predictors of depression, including biological predisposition, tendency of pessimism, traumatic experience, low selfesteem, and pressure. Other researchers found that Intolerance of Uncertainty (IU) and emotional regulation (ER) are two essential elements for the development and maintenance of negative emotional status in the majority of the population [3-6] (Aldao et al., 2016; Carleton, 2016; Gross \& John, 2003; McEvoy \& Mahoney, 2012). In recent years, Bunting (2016) pointed out that mindfulness could be utilized as a treatment for depression [7] (Bunting, 2016), and the possibility that low levels of mindfulness could be a contributing factor to the development of depression.

To date, a few studies have investigated depression from a cultural perspective. For instance, Zhang (1995) explained the lower rates of depression in China through different uses of traditional medicine, political norms, and ways of expressing emotion linguistically [8]. Other studies have suggested that the rate of depression diagnosis is low in China because these same symptoms are instead being diagnosed as neurasthenia, which is no longer included in the DSM-IV [9-12] (Cheung, 1982, 1984; Kleinman, 1986; Yeh, 1987). Since the conceptualization and interpretation of depression are culturally defined [9][13] (Cheung, 1982; Nikelly, 1988), people in China are diagnosed as neurasthenic as long as their depressionrelated symptoms are not sufficiently severe [14] (Yan, 1985). However, no cross-cultural research has investigated other potential predictors of depression, an important direction considering the varied and unpredictable nature of depression. The factors we hope to investigate include intolerance of uncertainty (IU), mindfulness, and stress.

\subsection{Intolerance of Uncertainty (IU)}

The negative feeling that accompanies the belief that there is no way to predict the occurrences or outcomes of future events has been characterized as intolerance of uncertainty (IU). People who are intolerant of uncertainty are much more likely to regard ambiguous situations as threatening [15] (Heydayati, Dugas, Buhr, \& Francis, 2003). Most recently, Carleton (2016) has defined IU as the inability to endure negative emotions caused by the lack of what one considers key information and substantiated by the perception of uncertainty [4]. IU has been shown to be correlated with major depressive disorder [16-20] (MDD; de Jong-Meyer et al. 2009; Gentes and Ruscio 2011; McEvoy and Mahoney 2012; Miranda et al. 2008; Nelson et al. 2014), social anxiety [21-22] (Boelen and Reijntjes 2009; Teale Sapach et al. 2015), and obsessive 
compulsive disorder [23-25] (OCD; Lind and Boschen 2009; Steketee et al. 1998; Tolin et al. 2003).

Cultural differences in uncertainty avoidance is one of Hofstede's five cultural dimensions which consist of power distance, uncertainty avoidance, masculinityfemininity, long-term versus short-term orientation, and individualism-collectivism [26] (Hofstede, 2001). Specifically, uncertainty avoidance was depicted as the extent to which people of a culture avoid or tolerate uncertain and ambiguous situations [26] (Hofstede, 2001). Hofstede (2001) pointed out that people from high uncertainty avoidance cultures demonstrate higher levels of anxiety [26]. Findings from another study pointed out that high uncertainty avoidance and masculinityfemininity predicts higher national levels of agoraphobic fears, fears of illness and death, and fears of sexual and aggressive scenes [27] (Arrindell et al., 2004). Since we expected anxiety and fear to be closely tied to depression, we suspected that uncertainty avoidance could be a predictor in explaining the differences in depression rates across various countries. Given the definition of uncertainty avoidance is highly similar to IU and it has been shown to consistently affect mental health, in this study, we investigated whether IU is a mechanism underlying cultural differences in depression rates.

\subsection{Mindfulness}

Mindfulness is an ancient practice from Eastern spiritual traditions [28-29] (Hick, 2008; Shapiro \& Carlson, 2009), which has been broadly defined as being mindful of one's arising thoughts, feelings, and sensations in a nonjudgmental and person-centered manner. One is considered to be experiencing mindfulness when thoughts, feelings, and sensations generated in one's attention field are noticed and accepted as they are [30] (Segal et al., 2002). In recent decades, an increasing number of mindfulness training programs has been documented by researchers [31] (Salmon, Santorelli, \& Kabat-Zinn, 1998). The main goal of mindfulness-based programs is to increase awareness of how habitual cognitive reactivity to sensations, feelings, and thoughts increase stress and distressing emotions, and to alleviate the vulnerability to these negative reactions, thereby generating persistent improvements in mental well-being [32] (Teasdale, 1999). Teasdale and colleagues pointed out that mindfulnessbased interventions are able to substantially decrease a range of psychological conditions, including depressive relapse [32]. Hofmann and colleagues (2010) also indicated in their study that mindfulness-based therapy (MBT) can be effective in mitigating acute symptoms of depression and anxiety [33].

To date, no research has directly measured whether national levels of mindfulness differ across various countries. However, studies in recent years have shown an association between counselors' mindfulness levels and their competence in multicultural counseling: components of mindfulness are highly associated with multicultural attitude (the recognition of the effects of culture on people's lives) and multicultural knowledge [34] (seeking information from different cultures; Iver et al., 2014). Once again, however, since no research has examined whether levels of mindfulness in the general population relate to depression rates specifically, mindfulness will be another predictor that we will test in our analyses.

Stress has been defined in a range of ways. Kennette and Lin (2019) found that stress impedes productivity and job satisfaction while at the same time leading to poorer performance across a variety of industries [35]. Similarly, Felver, Morton, and Clawson's (2018) study focused on college students presenting with academic, personal growth, and social development stressors and found that they were at increased risk of psychological distress [36] On the other hand, Baghurst and Kelly (2014) concluded that stress is unavoidable and that its triggers are diverse [37]. Deasy and colleagues (2014) warned that stress can result in poor coping behaviors and unhealthy habits, which could then circle around to increase stress, thereby perpetuating a vicious cycle [38].

\subsection{Stress}

A few researchers have assessed sources and mediators of stress from a multicultural perspective. For instance, Defunk, Ivancevich, and Schweiger (1988) found that Japanese managers are more likely to report more stress, less social support, less job satisfaction, and more negative personal characteristics compared to U.S. and Indian managers [39]. In addition, Radford and colleagues (1993) examined the effect of culture on decisional selfesteem, decisional stress, and decision coping styles in both Japanese and Australian university students. They found that Japanese students reported lower decisional self-esteem and higher decisional stress. The authors concluded that these differences were due to cultura differences in individualism (higher in Australia) and collectivism (higher in Japan) [40].

\section{METHOD}

\subsection{Participants}

A power analysis based on previous studies showed that in order to detect the largest effect size found in these studies $(\mathrm{d}=0.25)$ with $95 \%$ power, we would need 50 participants in each sample. Therefore, we recruited 50 US American participants from Amazon Mechanical Turk and 50 Chinese participants from the Chinese WenJuan Website. We ensured that participants accurately represent our intended cultural samples by requiring US Americans to: (a) be born in the US, (b) be living in the US, and (c) have parents who were born in the US. Similarly, Chinese participants were required to: (a) be born in China, (b) be living in China, and (c) have parents who were born in China. After excluding participants who did not fit these 
criteria, we were left with a final sample of 47 US American participants and 44 Chinese participants. US American participants had a mean age of 40 years $(\mathrm{SD}=$ 11.9), whereas Chinese participants had a mean age of 30 $(\mathrm{SD}=10.5) .49 \%$ of the US American sample was female, whereas $55 \%$ of the Chinese sample was female. Since the two samples differed significantly on several demographic variables listed above, we entered age and gender as covariates in all of the analyses presented here.

\subsection{Research Design}

To test whether intolerance of uncertainty, mindfulness, and stress mediated the cultural difference in depression between US Americans and Chinese, we conducted a mediation analysis on all three variables. For all of the analyses listed above, we also controlled for age and gender.

\subsection{Procedure}

Participants were informed that they would be completing a survey about their thoughts and feelings that would last about 7 10 minutes. US American participants were compensated $\$ 1.20$ (along with the federal minimum wage rate), while Chinese participants were compensated with 5 RMB. All measures were translated (and backtranslated) into Chinese.

\subsubsection{Intolerance of Uncertainty Scale}

Intolerance of uncertainty was measured using the short version of the Intolerance of Uncertainty Scale (IUS), which is a 12-item two-factor version of IUS [41] (IUS12; Carleton, Norton, \& Asmundson, 2007). This shortened version of the scale was shown to be highly correlated with the original IUS (IUS; Freeston, Rheaume, Letarte, Dugas, \& Ladouceur, 1994). Participants were asked to read each question and "select the number that best corresponds to how much you agree with each question" on a 1-5 Likert scale $(1=$ Not at all characteristic of me, $5=$ Entirely characteristic of me). Cronbach's alpha for this scale was 0.90 .

\subsubsection{Mindfulness}

Mindfulness was measured using the 13-item Toronto Mindfulness Scale. Participants were asked to "Read each statement and indicate the extent to which you agree with each statement" on a 0-4 Likert scale $(0=$ Not at all, $4=$ Very much). The scale was broken down into two subscales: Curiosity (e.g. I was curious about what I might learn about myself by taking notice of how I react to certain thoughts, feelings or sensations) and Decentering (e.g. I experience myself as separate from my changing thoughts and feelings). Each participant's mindfulness score was computed based on their average score across all items. Cronbach's alpha for this scale was 0.89 .

\subsubsection{Depression/Anxiety/Stress}

The 42-item Depression Anxiety Stress Scale (DASS) was used to measure depression, anxiety, and stress. Participants were asked to "read each statement and select a number $0,1,2$ or 3 which indicates how much the statement applie[s] to [them] over the past week." They were reminded that there were no right or wrong answers and to not take too much time on any statement. Participants answered on a 0-3 Likert scale $(0=$ Did not apply to me at all, $3=$ Applied to me very much, or most of the time). Cronbach's alpha for the Depression subscale was 0.97 ; the Anxiety subscale was 0.90 ; and the Stress subscale was 0.96 .

\section{RESULTS}

\subsection{Cultural Differences in Depression}

First, we wanted to replicate the finding that Americans have a higher rate of depression than Chinese (WHO, 2004). As expected, a multiple regression analysis revealed that US American participants scored higher on the DASS scale than their Chinese counterparts, $\mathrm{F}(3,87)=$ $5.46, \mathrm{p}<.001, \mathrm{R} 2=.16$.

\subsection{Cultural Differences in Stress, Intolerance of Uncertainty, and Mindfulness}

Before running our mediational analyses, we looked at whether there were cultural differences in our hypothesized mediational variables. We found that there was indeed a cultural difference in stress, such that US Americans reported higher stress than their Chinese counterparts $\mathrm{F}(3,87)=10.23, \mathrm{p}<.001, \mathrm{R} 2=.26$. However, since we did not find a significant cultural difference in intolerance of uncertainty $(\mathrm{F}(3,87)=1.25$, p $=.30, \mathrm{R} 2=.04)$ nor mindfulness $(\mathrm{F}(3,87)=2.49, \mathrm{p}=.07$, $\mathrm{R} 2=.08)$, we were not able to proceed with the mediational analyses.

\subsection{Mediators of Group Differences in Depression}

We hypothesized that stress, mindfulness, and IU may mediate the cultural difference in depression. To test this hypothesis, we conducted a series of multiple regressions to evaluate whether the four criteria for mediation were met [42] (Baron \& Kenny, 1986; Frazier, Tix, \& Barron, 2004), using the R package Mediation. Specifically, we 
examined whether (a) the independent variable (Group, US American participants $=0$, Chinese participants $=1$ ) was significantly correlated with the outcome variable (depression), (b) the independent variable (Group) was significantly correlated with the potential mediator (stress, mindfulness, IU), (c) the potential mediator (stress, mindfulness, IU) was significantly correlated with the outcome variable (depression), controlling for the independent variable (Group), and (d) the mediational effect was significant according to the Sobel test. Separate analyses were conducted for each potential mediator. To control for differences in age and gender across the two cultural groups, we treated age and gender as covariates in each meditation.

The first criterion for mediation was met: Group was correlated with depression $(\beta=-0.44, \mathrm{SE}=0.21, \mathrm{~B}=$ $0.85, \mathrm{t}=-4.05, \mathrm{p}<.001)$. The second criterion was met for stress $(\beta=-0.55, \mathrm{SE}=0.19, \mathrm{~B}=-1.07, \mathrm{t}=-5.48, \mathrm{p}<$ $.001)$, but not for mindfulness $(\beta=0.20, \mathrm{SE}=0.18, \mathrm{~B}=$ $0.33, \mathrm{t}=1.78, \mathrm{p}=.08)$ nor IU $(\beta=0.11, \mathrm{SE}=0.24, \mathrm{~B}=$ $0.24, \mathrm{t}=0.97, \mathrm{p}=.33)$. The third criterion for mediation was met for stress $(\beta=0.72, \mathrm{SE}=0.75, \mathrm{~B}=0.73, \mathrm{t}=9.66$, $\mathrm{p}<.001)$. As Figure 1 illustrates, based on nonparametric bootstrap estimation approach with 500 samples, we found that the bootstrapped indirect effect was -.75 and the $95 \%$ confidence interval ranged from $[-1.11,-0.43]$. The indirect effect was statistically significant $(\mathrm{p}<.001)$.

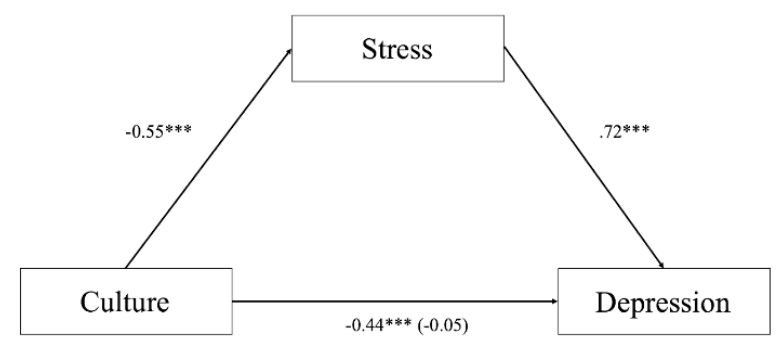

Figure 1 indicated the standardized regression coefficients for the cultural difference in depression as mediated by stress. The standardized regression coefficient for the cultural differences in depression, controlling for stress, is in parentheses.

\section{DISSCUSSION}

In this study, we tested intolerance of uncertainty (IU), mindfulness, and stress as potential mediators of the cultural difference in depression between US American and Chinese participants. US American participants, compared to their Chinese counterparts, reported higher levels of both depression and stress. We found that stress was strongly and positively related to depression for both cultural groups. In contrast, we found no cultural difference in IU nor mindfulness. Therefore, we concluded that stress fully mediates the cultural differences in depression.
A few studies have examined the association between stress and job satisfaction [39] (Defunk et al., 1988) as well as stress and self-esteem [40] (Radford et al., 1993) from a cross-cultural perspective. These studies indicate that stress is more likely to negatively affect one's sense of job satisfaction and self-concept. In this study, we found that stress predicts negative emotional reactions (depression) across both US American and Chinese cultures, which is in line with prior research. Since not much cross-cultural work has been conducted to investigate the association between specific factors and depression, the aim of our study was to figure out what mechanism drives cultural differences in depression.

There are several limitations worth discussing in this study. Firstly, due to the fact that we had to exclude multiple participants who did not pass our attention checks, the sample size was limited, potentially lowering our power to detect cultural differences. In particular, it is possible that given adequate power we may have detected a significant difference in mindfulness, especially since with our sample we achieved marginal significance. In addition, it is possible that there were important demographic differences between our cultural samples. Specifically, in our samples, Chinese participants were younger than their US American counterparts. While we did not see any other demographic differences between the cultural samples, it is possible that they differed on a dimension that we did not measure in this study.

Another limitation is the fact that we did not randomly assign participants to US American or Chinese culture, and therefore are unable to assess to what extent culture is the causal factor. In addition, since stress and depression are correlational, one alternate interpretation of our results is that the differences in depression levels are driving the differences in stress. We have not found out the direction of the causal arrow between stress and depression in different cultures. Last but not least, the predictors for depression are varied and complicated. In this study we did not measure other potential components. For instance, emotion regulation has been shown to be an essential process related to a wide range of mood disorders [3] (Aldao et al., 2016), including major depressive disorder. Moreover, Liao and Wei (2011) have examined rumination as a mediator and moderator between IU and depression symptoms, and they concluded that rumination is a full mediator between IU and depression [43]. Had we measured emotion regulation and rumination, for example, it is possible that we would have a richer picture of what is going on.

Our study's findings suggest some future research directions. Firstly, future research could include additional predictors and mediators to explain the cultural differences in depression, as well as other mental health issues. Second, future studies could investigate depression in populations from other cultures to detect additional explanatory factors. In addition, due to the complexity of mental health issues, very few researches have explored the causal mechanism linking various predictors with depression. Future research could further isolate the various components of depression to study how each is 
linked to mental health in different cultural contexts. Finally, in this study, we focused on the mechanism driving the cultural difference in depression, but it will be important for future research to investigate the specific protective and risk factors that can then potentially inform culture-specific interventions.

\section{CONCLUSION}

In conclusion, our study finds that stress fully explains the cultural differences in depression between the US American and Chinese participants. The current results have practical clinical implications. Since stress is highly correlated with depression in both cultures, it is more likely that it plays a part in one's mental health status. Our results suggest that counselors and therapists should become more aware of the important role of stress in depression, and the results put more attention on developing effective coping skills to reduce stress.

\section{REFERENCES}

[1] Johnson, P. R., \& Indvik, J. (1997). The boomer blues: Depression in the workplace. Public Personnel Management, 26(3), 359.

[2] World Health Organization. (2004, February). Agestandardized DALYs per 100,000 by cause, and Member State.

[3] Aldao, A., Gee, D. G., De Los Reyes, A., \& Seager, I. (2016). Emotion regulation as a transdiagnostic factor in the development of internalizing and externalizing psychopathology: Current and future directions. Development and Psychopathology, 28(4pt1), 927-946.

[4] Carleton, R. N. (2016). Into the unknown: A review and synthesis of contemporary models involving uncertainty. Journal of Anxiety Disorders, 39, 30-43.

[5] Gross, J. J., \& John, O. P. (2003). Individual differences in two emotion regulation processes: Implications for affect, relationships, and well-being. Journal of Personality and Social Psychology, 85(2), 348362 .

[6] McEvoy, P. M., \& Mahoney, A. E. (2012). To be sure, to be sure: Intolerance of uncertainty mediates symptoms of various anxiety disorders and depression. Behavioral Therapy, 43(3), 533-545.

[7] Bunting, M. (2016). Why mind matters. Resurgence \& Ecologist, (294), 26-29.

[8] Zhang, D. (2007). Depression and Culture --- A Chinese Perspective. Canadian Journal of Counselling and Psychotherapy, 29(3).
[9] Cheung, F. M. (1982). Psychological symptoms among Chinese in urban Hong Kong. Social Science and Medicine, 16, 1339-44.

[10] Cheung, F. M. (1984). Preferences in help-seeking among Chinese students. Culture, Medicine and Psychiatry, 8, 371-80.

[11] Kleinman, A. (1986). Social origins of distress and disease-depression, neurasthenia, and pain in modern China. New Haven: Yale University Press.

[12] Yeh, E. K. (1987). Social changes and prevalence of specific mental disorders in Taiwan. Chinese Journal of Mental Health, 3, 31-42.

[13] Nikelly, A. G. (1988). Does DSM-III-R diagnose depression innon-Western patients? International Journal of Social Psychiatry, 34, 316-20

[14] Yan, H. Q. (1985). Some psychological problems manifested by neurotic patients: Shanghai examples. In: W. S. Tseng \& D. YH. Wu (eds.), Chinese Culture and Mental Health. New York: Wiley. pp. 325-337

[15] Heydayati, M., Dugas, M. J., Buhr, K., \& Francis, K. (2003, November). The relationship between intolerance of uncertainty and the interpretation of ambiguous and unambiguous information. Poster presented at the Annual Convention of the Association for Advancement of Behavior Therapy, Boston, MA.

[16] de Jong-Meyer, R., Beck, B., \& Riede, K. (2009). Relationships between rumination, worry, intolerance of uncertainty and mega-cognitive beliefs. Personality and Individual Differences, 46(4), 547-551.

[17] Gentes, E. L., \& Ruscio, A. M. (2011). A metaanalysis of the relation of intolerance of uncertainty to symptoms of generalized anxiety disorder, major depressive disorder, and obsessive-compulsive disorder. Clinical Psychology Review, 31(6), 923-933.

[18] McEvoy, P. M., \& Mahoney, A. E. (2012). To be sure, to be sure: Intolerance of uncertainty mediates symptoms of various anxiety disorders and depression. Behavioral Therapy, 43(3), 533-545.

[19] Miranda, R., Fontes, M., \& Marroquin, B. (2008). Cognitive content- specificity in future experiences: Role of hopelessness and intolerance of uncertainty in depression and GAD symptoms. Behavior Research and Therapy, 46(10), 1151-1159.

[20] Nelson, B. D., Shankman, S. A., \& Proudfit, G. H (2014). Intolerance of uncertainty mediates reduced reward anticipation in major depressive disorder. Journal of Affective Disorders, 158, 108-113. 
[21] Boelen, P. A., \& Reijntjes, A. (2009). Intolerance of uncertainty and social anxiety. Journal of Anxiety Disorders, 23(1), 130-135.

[22] Teale Sapach, M. J., Carleton, R. N., Mulvogue, M. K., Weeks, J. W., \& Heimberg, R. G. (2015). Cognitive constructs and social anxiety disorder: Beyond fearing negative evaluation. Cognitive Behavior Therapy, 44(1), 63-73.

[23] Lind, C., \& Boschen, M. J. (2009). Intolerance of uncertainty mediates the relationship between responsibility beliefs and compulsive checking. Journal of Anxiety Disorders, 23(8), 1047-1052.

[24] Steketee, G., Frost, R. O., \& Cohen, I. (1998). Beliefs in obsessive-compulsive disorder. Journal of Anxiety Disorders, 12(6), 525-537.

[25] Tolin, D. F., Abramowitz, J. S., Brigidi, B. D., \& Foa, E. B. (2003). Intolerance of uncertainty in obsessivecompulsive disorder. Journal of Anxiety Disorders, 17(2), 233-242.

[26] Hofstede, G. (2001). Culture's consequences: comparing values, behaviors, institutions, and organizations across nations (2nd ed.). Thousand Oaks, CA: Sage.

[27] Arrindell WA., Eisemann M, Oei TPS., et al. Phobic anxiety in 11 nations: part II. Hofstede's dimensions of national cultures predict national-level variations. Personality \& Individual Differences. 2004; 37(3), 627643.

[28] Hick, S. F. (2008). Cultivating therapeutic relationships: The role of mindfulness. In: S. Hick \& T. Bien (Eds.), Mindfulness and the therapeutic relationship. New York, NY: Guilford Press. pp. 3-18

[29] Shapiro, S. L., \& Carlson, L. E. (2009). The art and science of mindfulness: Integrating mindfulness into psychology and the helping professions. In: American Psychological Association. Washington, DC

[30] Segal Z. V., Teasdale J. D., Williams J. M., \& Gemar M. C. (2002). The Mindfulness-Based Cognitive Therapy Adherence Scale: Interrater reliability, adherence to protocol and treatment distinctiveness. Clinical Psychology and Psychotherapy, 9, 131-138.

[31] Salmon, P., Santorelli, S. F., and Kabat-Zinn, J. (1998). Inter- vention elements promoting high adherence to mindfulness- based stress reduction programs in the clinical behavioral medicine setting. In: S. A. Shumaker, E. B. Schron, J. K. Ockene, \& W. L. McBee (Eds.), Handbook of health behavior change. New York: Springer. pp. 239-266
[32] Teasdale, J. D. (1999). Metacognition, mindfulness and the modification of mood disorders. Clinical Psychology and Psychotherapy, 6, 146-155.

[33] Hofmann, S., Sawyer, A., Witt, A., \& Oh, D. (2011). The effect of mindfulness-based therapy on anxiety and depression: a meta-analytic review. Primary Health Care, 21(3), 14-14.

[34] Ivers, N. N., Johnson, D. A., Clarke, P. B., Newsome, D. W., \& Berry, R. A. (2016). The Relationship Between Mindfulness and Multicultural Counseling Competence. Journal of Counseling \& Development, 94(1), 72-82.

[35] Kennette, L. N., \& Lin, P. S. (2019). Focusing on Faculty Stress. Transformative Dialogues: Teaching \& Learning Journal, 12(1), 1-4.

[36] Felver, J. C., Morton, M. L., \& Clawson, A. J. (2018). Mindfulness-Based Stress Reduction Reduces Psychological Distress in College Students. College Student Journal, 52(3), 291-298.

[37] Baghurst, T., \& Kelley, B. C. (2014). An examination of stress in college students over the course of a semester. Health Promotion Practice, 15(3), 438-447.

[38] Deasy, C., Coughlan, B., Pironom, J., Jourdan, D., \& Mcnamara, P. M. (2015). Psychological distress and lifestyle of students: Implications for health promotion. Health Promotion International, 30(1), 77-87.

[39] Defunk, R., Ivancevich, J., \& Schweiger, D. (1988). Job Stress and Mental Well-Being: Similarities and Differences among American, Japanese, and Indian Managers. Behavioral Medicine, 14(4), 160-170.

[40] Radford, M. H. B., Mann, L., Ohta, Y., \& Nakane, Y. (1993). Differences between Australian and Japanese Students in Decisional Self-Esteem, Decisional Stress, and Coping Styles. Journal of Cross-Cultural Psychology, 24(3), 284-297.

[41] Carleton RN, Norton MA, Asmundson GJ. Fearing the unknown: a short version of the Intolerance of Uncertainty Scale. Journal of Anxiety Disorders. 2007 ;21(1):105-117.

[42] Baron, R., \& Kenny, D. (1986). The moderatormediator variable distinction in social psychological research: Conceptual, strategic, and statistical considerations. Journal of Personality and Social Psychology, 51(6), 1173-1182.

[43] Liao, K. Y., \& Wei, M. (2011). Intolerance of uncertainty, depression, and anxiety: the moderating and mediating roles of rumination. Journal of Clinical Psychology, 67(12), 1220-1239. 